\title{
Synthesis and characterization of TADDOL-based chiral group six PNP pincer tricarbonyl complexes
}

\author{
Sara R. M. M. de Aguiar ${ }^{1} \cdot$ Christian Schröder-Holzhacker $^{1} \cdot$ Jan Pecak $^{1} \cdot$ Berthold Stöger $^{2}$. \\ Karl Kirchner ${ }^{1}$
}

Received: 17 June 2018 / Accepted: 14 August 2018/Published online: 20 October 2018

(C) The Author(s) 2018

\begin{abstract}
The new chiral PNP pincer ligand $N^{2}, N^{6}$-bis $((3 \mathrm{a} R, 8 \mathrm{a} R)-2,2$-dimethyl-4,4,8,8-tetraphenyltetrahydro[1,3]dioxolo[4,5$e][1,3,2]$ dioxaphosphepin-6-yl)pyridine-2,6-diamine (PNP-TADDOL) was synthesized in 80\% isolated yield. Complexes of the type $\left[\mathrm{M}(\mathrm{PNP}-\mathrm{TADDOL})(\mathrm{CO})_{3}\right](\mathrm{M}=\mathrm{Cr}$, Mo, and $\mathrm{W})$ were prepared via a solvothermal approach. This methodology constitutes a fast, simple, and practical synthetic method to obtain complexes of that type in high isolated yields. The $\mathrm{X}$-ray structure of the molybdenum complex is presented.
\end{abstract}

\section{Graphical abstract}
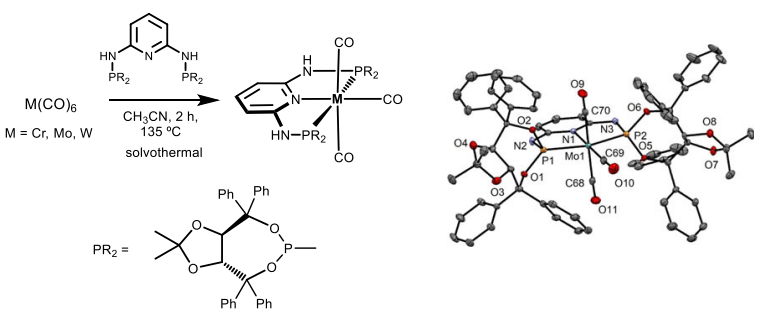

Keywords Chiral pincer ligands · Group six metals - Solvothermal synthesis - TADDOL · Carbonyl ligands

\section{Introduction}

PNP pincer ligands with a pyridine backbone and phosphorus donors connected in the two ortho position via $\mathrm{CH}_{2}$, $\mathrm{O}$, NH, or NR spacers are widely utilized in transition metal chemistry. They form often very stable but also reactive complexes which can be designed in modular fashion. They typically adopt a meridional coordination mode via the two electron donor groups and a metal-

Karl Kirchner

karl.kirchner@tuwien.ac.at

1 Institute of Applied Synthetic Chemistry, Vienna University of Technology, Getreidemarkt 9, 1060 Vienna, Austria

2 X-ray Center, Vienna University of Technology, Getreidemarkt 9, 1060 Vienna, Austria nitrogen bond. Over the last decades, pincer complexes have found numerous applications in various areas of chemistry, especially organic synthesis and catalysis [1-12].

As group six PNP complexes are concerned, such compounds are exceedingly rare and only a few examples have been reported in the literature [13-26]. We are currently focusing on the synthesis and reactivity of group six complexes containing PNP pincer ligands based on the 2,6diaminopyridine scaffold. In these ligands, the aromatic pyridine ring and the phosphine moieties are connected via $\mathrm{NH}, \mathrm{N}$-alkyl, or $\mathrm{N}$-aryl spacers [27-31]. Such ligands were first utilized by the group of Haupt who prepared PNP pincer complexes of the type $\left[\mathrm{M}(\mathrm{PNP}-\mathrm{Ph})(\mathrm{CO})_{3}\right](\mathrm{M}=\mathrm{Cr}$, Mo, $\quad$ W; $\quad \mathrm{PNP}-\mathrm{Ph}=N^{2}, N^{6}$-bis(diphenylphosphanyl) pyridine-2,6-diamine) [13]. In continuation of our studies 
on group six PNP complexes, we report here the synthesis of chiral zero valent $\mathrm{Cr}$, Mo, and W PNP pincer complexes based on $((4 R, 5 R)-2,2$-dimethyl-1,3-dioxolane-4,5diyl)bis(diphenylmethanol) (R,R-TADDOL). It has to be mentioned that chiral pincer complexes are comparatively rare [32-34].

\section{Results and discussion}

The new $\mathrm{C}_{2}$-symmetric chiral pincer ligand $N^{2}, N^{6}$ bis((3aR,8aR)-2,2-dimethyl-4,4,8,8-tetraphenyltetrahydro[1,3]dioxolo[4,5-e][1,3,2]dioxaphosphepin-6-yl)pyridine-2,6diamine (PNP-TADDOL, 2) was obtained in $80 \%$ isolated yield by reacting 2,6-diaminopyridine with 2 equiv. of 1 in the presence of $\mathrm{NEt}_{3}$ as base as shown in Scheme 1. The pincer ligand is air-stable and was characterized by ${ }^{1} \mathrm{H},{ }^{13} \mathrm{C}\left\{{ }^{1} \mathrm{H}\right\}$, and ${ }^{31} \mathrm{P}\left\{{ }^{1} \mathrm{H}\right\}$ NMR spectroscopy.
A suspension of the hexacarbonyl complexes $\mathrm{M}(\mathrm{CO})_{6}$ $(\mathrm{M}=\mathrm{Cr}, \mathrm{Mo}, \mathrm{W})$ and the PNP ligand 2 in $\mathrm{CH}_{3} \mathrm{CN}$ were placed in a sealed microwave glass vial and stirred for $2 \mathrm{~h}$ at $135^{\circ} \mathrm{C}$. After workup, the analytically pure products $\left[\mathrm{M}(\mathrm{PNP}-\mathrm{TADDOL})(\mathrm{CO})_{3}\right]$ 3-5 could be isolated in $89-91 \%$ yields (Scheme 2). All complexes are air stable. Moreover, in the presence of small amounts of water, $\mathrm{P}-\mathrm{N}$ and/or $\mathrm{P}-\mathrm{O}$ bond cleavage as a result of hydrolysis was not observed.

All complexes were fully characterized by a combination of ${ }^{1} \mathrm{H},{ }^{13} \mathrm{C}\left\{{ }^{1} \mathrm{H}\right\}$, and ${ }^{31} \mathrm{P}\left\{{ }^{1} \mathrm{H}\right\}$ NMR spectroscopy, IR spectroscopy, and elemental analysis. In the ${ }^{13} \mathrm{C}\left\{{ }^{1} \mathrm{H}\right\} \mathrm{NMR}$ spectrum, these complexes exhibit two characteristic lowfield triplet resonances in a 1:2 ratio in the range of 228-206 ppm assignable to the carbonyl carbon atoms trans and $c i s$ to the pyridine nitrogen atom, respectively (Table 1). The ${ }^{31} \mathrm{P}\left\{{ }^{1} \mathrm{H}\right\}$ NMR spectra exhibit singlet resonances in the range of 198-153 ppm. In the case of the tungsten complex, the spectra exhibit singlet resonances with ${ }^{1} J_{\mathrm{WP}}$ coupling constants of $499 \mathrm{~Hz}$. The tungsten-phosphorus coupling was

Scheme 1<smiles>CC1(C)O[C@H]2[C@H](O1)C(c1ccccc1)(c1ccccc1)OP(Cl)OC2(c1ccccc1)c1ccccc1</smiles><smiles>CCOCCN</smiles><smiles>PNc1cccc(NP)n1</smiles>

$\mathrm{PR}_{2}=$<smiles>CP1OC(c2ccccc2)(c2ccccc2)[C@H]2OC(C)(C)O[C@@H]2C(c2ccccc2)(c2ccccc2)O1</smiles>

Scheme 2

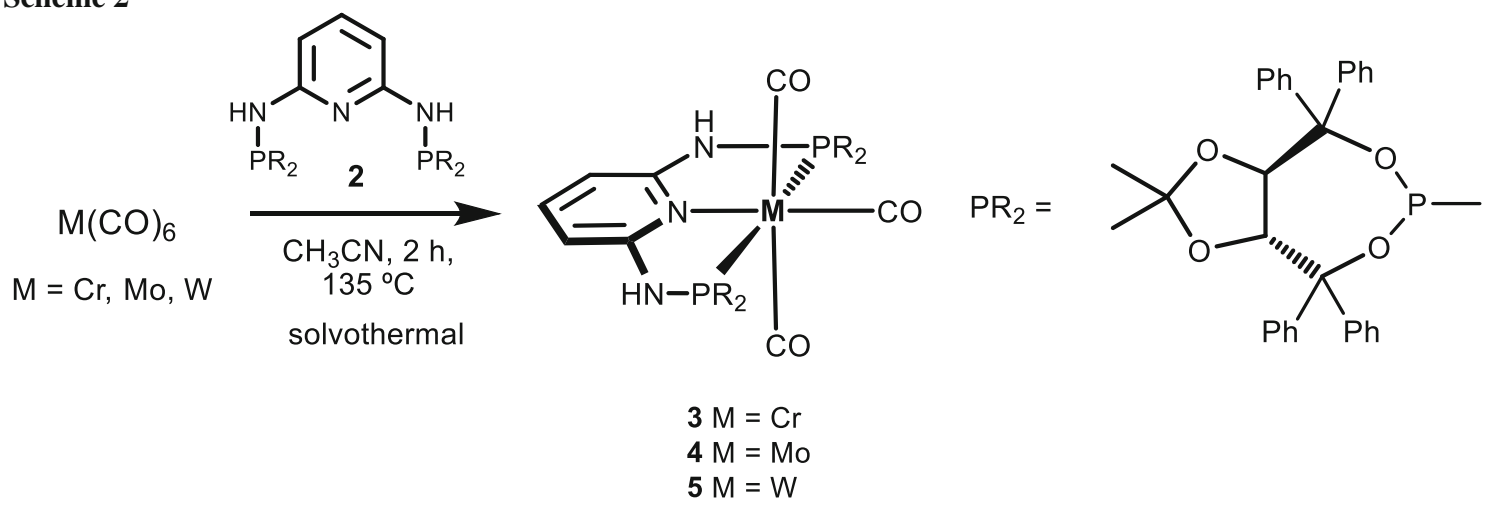


Table 1 Selected ${ }^{13} \mathrm{C}\left\{{ }^{1} \mathrm{H}\right\}$ and ${ }^{31} \mathrm{P}\left\{{ }^{1} \mathrm{H}\right\}$ NMR and IR data of complexes 3-5 and related molybdenum tricarbonyl complexes [13, 15, 16]

\begin{tabular}{lllllll}
\hline Complexes & $\delta_{\mathrm{CO}} / \mathrm{ppm}$ & & $\delta_{\mathrm{P}} / \mathrm{ppm}$ & $\delta_{\mathrm{CO}} / \mathrm{cm}^{-1}$ & & \\
\hline$\left[\mathrm{Cr}(\mathrm{PNP}-\mathrm{TADDOL})(\mathrm{CO})_{3}\right](\mathbf{3})$ & 228.0 & 216.9 & 197.8 & 1974 & 1921 & 1865 \\
{$\left[\mathrm{Mo}(\mathrm{PNP}-\mathrm{TADDOL})(\mathrm{CO})_{3}\right](\mathbf{4})$} & 221.6 & 206.7 & 172.7 & 1980 & 1946 & 1867 \\
{$\left[\mathrm{~W}(\mathrm{PNP}-\mathrm{TADDOL})(\mathrm{CO})_{3}\right](\mathbf{5})$} & 214.3 & 201.0 & 153.8 & 1978 & 1932 & 1865 \\
{$\left[\mathrm{Mo}(\mathrm{PNP}-\mathrm{BIPOL})(\mathrm{CO})_{3}\right]$} & 224.7 & 208.4 & 204.8 & 1985 & 1876 & - \\
{$\left[\mathrm{Mo}(\mathrm{PNP}-\mathrm{Ph})(\mathrm{CO})_{3}\right]$} & 228.4 & 211.2 & 104.0 & 1964 & 1858 & 1765 \\
{$\left[\mathrm{Mo}\left(\mathrm{PNP}^{\mathrm{Me}}-\mathrm{Ph}\right)(\mathrm{CO})_{3}\right]$} & 227.8 & 211.9 & 131.0 & 1956 & 1911 & 1850 \\
{$\left[\mathrm{Mo}\left(\mathrm{PNP}{ }^{\mathrm{Me}}-\mathrm{Et}\right)(\mathrm{CO})_{3}\right]$} & 230.6 & 214.9 & 132.3 & 1942 & 1822 & 1806 \\
{$\left[\mathrm{Mo}(\mathrm{PNP}-\mathrm{Cy})(\mathrm{CO})_{3}\right]$} & 231.1 & 216.4 & 122.6 & 1941 & 1828 & 1790 \\
{$\left[\mathrm{Mo}(\mathrm{PNP}-i \mathrm{Pr})(\mathrm{CO})_{3}\right]$} & 231.4 & 216.9 & 132.7 & 1936 & 1809 & 1790 \\
{$\left[\mathrm{Mo}\left(\mathrm{PNP}{ }_{-}^{\mathrm{Me}}-i \mathrm{Pr}\right)(\mathrm{CO})_{3}\right]$} & 230.8 & 217.9 & 159.0 & 1936 & 1810 & 1795 \\
{$\left[\mathrm{Mo}(\mathrm{PNP}-\mathrm{Et})(\mathrm{CO})_{3}\right]$} & 230.3 & 213.8 & 111.3 & 1929 & 1840 & 1780 \\
{$\left[\mathrm{Mo}(\mathrm{PNP}-t \mathrm{Bu})(\mathrm{CO})_{3}\right]$} & 233.1 & 224.0 & 148.8 & 1922 & 1808 & 1771 \\
\hline
\end{tabular}

Scheme 3<smiles>[R]N([R2])c1cccc(N([R2])[R])n1</smiles>

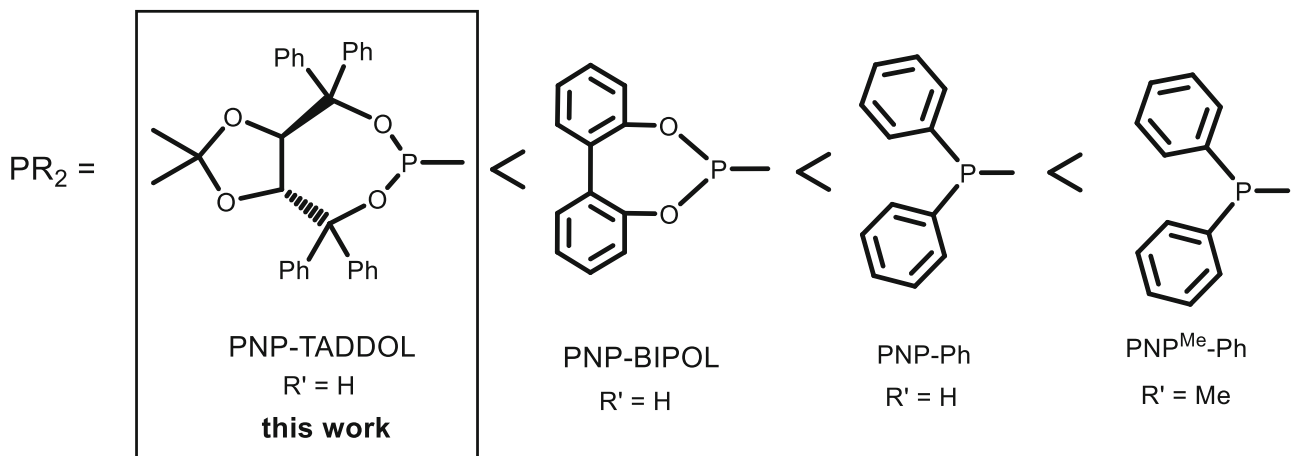
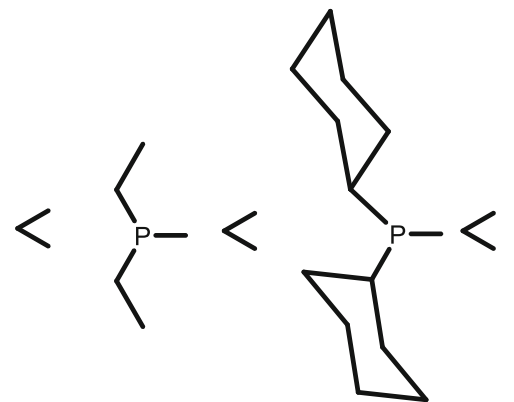

PNP-Et

$\mathrm{R}^{\prime}=\mathrm{Me}$

\begin{abstract}
PNP-Cy
\end{abstract}
$\mathrm{R}^{\prime}=\mathrm{H}$

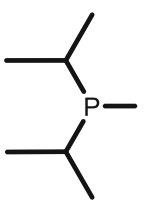

PNP-iPr

$\mathrm{R}^{\prime}=\mathrm{H}$
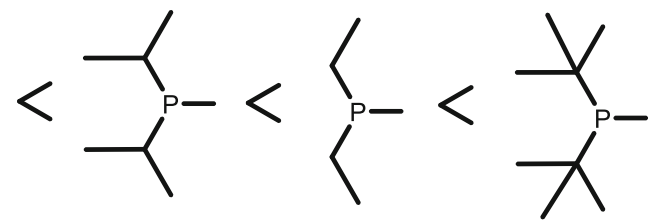

$\mathrm{PNP}^{\mathrm{Me}}-\mathrm{Pr}$

$\mathrm{R}^{\prime}=\mathrm{Me}$
PNP-Et

$\mathrm{R}^{\prime}=\mathrm{H}$
PNP-tBu

$\mathrm{R}^{\prime}=\mathrm{H}$ detected as a doublet satellite due to ${ }^{183} \mathrm{~W}$. This isotope has a $14 \%$ natural abundance with a spin $I$ of $1 / 2$. This signal is superimposed over the dominant singlet.

Both the carbonyl resonances $\left(\delta_{\mathrm{CO}}\right)$ and the phosphorus resonances $\left(\delta_{\mathrm{P}}\right)$ exhibit a significant upfield shift on going from $\mathrm{Cr}$ to $\mathrm{Mo}$ to $\mathrm{W}$. In all complexes, the PNP pincer ligand is coordinated in mer fashion with no evidence for any fac isomers. As expected of a mer $\mathrm{CO}$ arrangement, these complexes exhibit the typical three strong to medium absorption bands in the IR spectra. These are assignable to one weaker symmetric and two strong asymmetric vibrations. The $v_{\mathrm{CO}}$ frequencies, in particular the symmetric $\mathrm{CO}$ 


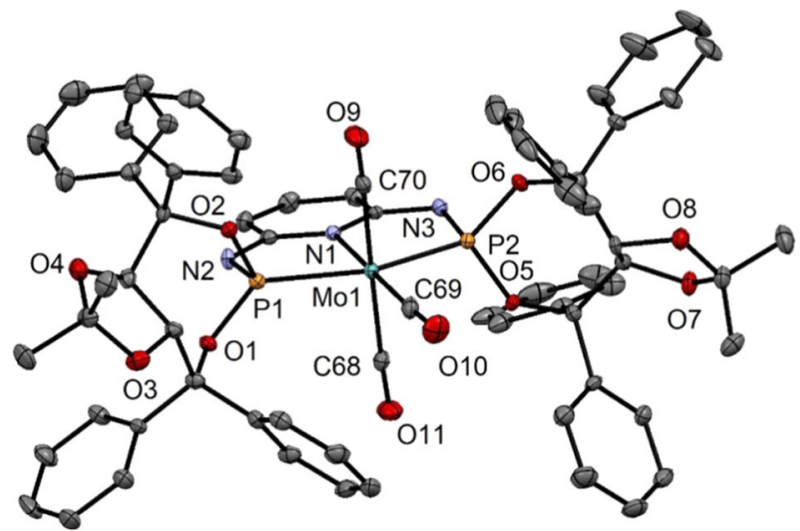

(a)

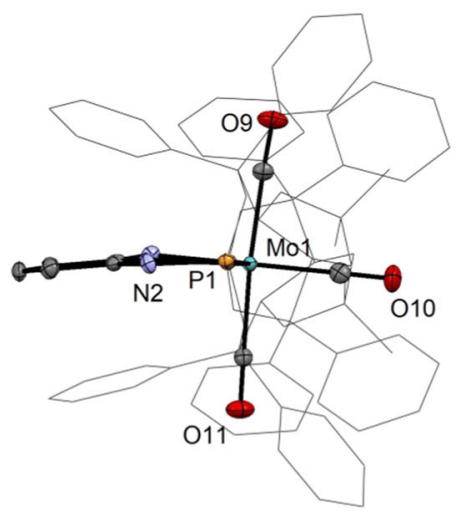

(b)
Fig. 1 a ORTEP plot of [Mo(PNP-TADDOL)(CO) $\left.)_{3}\right] \cdot 4 \mathrm{CH}_{2} \mathrm{Cl}_{2}$ $\left(4 \cdot 3 \mathrm{CH}_{2} \mathrm{Cl}_{2}\right)$ showing $50 \%$ thermal ellipsoids (hydrogen atoms and solvent omitted for clarity). b Side view of $4 \cdot 3 \mathrm{CH}_{2} \mathrm{Cl}_{2}$. Selected bond lengths $(\AA)$ and bond angles $\left({ }^{\circ}\right)$ : $\mathrm{Mo}_{1}-\mathrm{C}_{68}$ 2.029(6), $\mathrm{Mo}_{1}-\mathrm{C}_{69}$
1.967(6), $\mathrm{Mo}_{1}-\mathrm{C}_{70}$ 2.037(6), $\mathrm{Mo}_{1}-\mathrm{P}_{1}$ 2.329(1), $\mathrm{Mo}_{1}-\mathrm{P}_{2}$ 2.361(1), Mo- $\mathrm{N}_{1}$ 2.242(4), $\mathrm{P}_{1}-\mathrm{Mo}_{1}-\mathrm{P}_{2}$ 155.61(5), $\mathrm{N}_{1}-\mathrm{Mo}_{1}-\mathrm{C}_{69}$ 175.3(2), $\mathrm{C}_{68}-$ $\mathrm{Mo}_{1}-\mathrm{C}_{70} 175.8(2)$

\section{Conclusion}

In summary, we have prepared and fully characterized chiral group six metal complexes of the general formula $\left[\mathrm{M}(\mathrm{PNP}-\mathrm{TADDOL})(\mathrm{CO})_{3}\right](\mathrm{M}=\mathrm{Cr}, \mathrm{Mo}, \mathrm{W})$ bearing the $\mathrm{C}_{2}$-symmetric chiral TADDOL-based PNP pincer ligand with a pyridine backbone. Steric and electronic properties of these complexes could be directly compared with a series of other group six metal PNP pincer tricarbonyl complexes. Based on the symmetric $\mathrm{CO}$ stretch, which presents the highest stretching frequency, PNP-TADDOL is the weakest $\sigma$-donor, but the strongest $\pi$-acceptor of all PNP ligands in the series PNP-TADDOL $<$ PNPBIPOL $<$ PNP-Ph $<$ PNP $^{\mathrm{Me}}$-Ph $<\mathrm{PNP}^{\mathrm{Me}}$-Et $<$ PNP-Cy $<$ $\mathrm{PNP}-i \mathrm{Pr} \approx \mathrm{PNP}^{\mathrm{Me}}-i \mathrm{Pr}<\mathrm{PNP}-\mathrm{Et}<\mathrm{PNP}-t \mathrm{Bu}$. The X-ray structure of the molybdenum complex $[\mathrm{Mo}(\mathrm{PNP}-$ TADDOL $)(C O)_{3}$ ] is presented.

\section{Experimental}

All manipulations were performed under an inert atmosphere of argon using Schlenk techniques or in an MBraun inert-gas glovebox. The solvents were purified according to the standard procedures [35]. The deuterated solvents were purchased from Aldrich and dried over $4 \AA$ molecular sieves. The ligand precursor $(3 \mathrm{a} R, 8 \mathrm{a} R)-6$-chloro-2,2dimethyl-4,4,8,8-tetraphenyltetrahydro[1,3]dioxolo[4,5-e][1,3,2]dioxaphosphepine (TADDOL-PCl, 1) was prepared according to the literature [36]. ${ }^{1} \mathrm{H},{ }^{13} \mathrm{C}\left\{{ }^{1} \mathrm{H}\right\}$, and ${ }^{31} \mathrm{P}\left\{{ }^{1} \mathrm{H}\right\}$ NMR spectra were recorded on Bruker AVANCE-250, AVANCE-400 DRX, and AVANCE-600 spectrometers. ${ }^{1} \mathrm{H}$ 
and ${ }^{13} \mathrm{C}\left\{{ }^{1} \mathrm{H}\right\}$ NMR spectra were referenced internally to residual protio-solvent, and solvent resonances, respectively, and are reported relative to tetramethylsilane $(\delta=0 \mathrm{ppm})$. ${ }^{31} \mathrm{P}\left\{{ }^{1} \mathrm{H}\right\}$ NMR spectra were referenced externally to $\mathrm{H}_{3} \mathrm{PO}_{4}$ $(85 \%)(\delta=0 \mathrm{ppm})$.

\section{$N^{2}, N^{6}$-bis ((3aR,8aR)-2,2-dimethyl-4,4,8,8-tetraphenylte-} trahydro[1,3]dioxolo[4,5-e][1,3,2]dioxaphosphepin-6-yl)pyridine-2,6-diamine (PNP-TADDOL, 2, $\mathrm{C}_{67} \mathrm{H}_{61} \mathrm{~N}_{3} \mathrm{O}_{8} \mathrm{P}_{2}$ ) To a solution of $415 \mathrm{mg}$ 2,6-diaminopyridine $(4.11 \mathrm{mmol})$ in $100 \mathrm{~cm}^{3}$ toluene, $1.1 \mathrm{~cm}^{3} \mathrm{NEt}_{3}(10.27 \mathrm{mmol})$ was added. After cooling to $0{ }^{\circ} \mathrm{C}$, a solution of $4.58 \mathrm{~g} 1(8.63 \mathrm{mmol})$ in $30 \mathrm{~cm}^{3}$ of toluene was added and the reaction mixture was stirred for $12 \mathrm{~h}$ at $80{ }^{\circ} \mathrm{C}$. The suspension was filtered over Celite and the solvent was removed under reduced pressure. The product was obtained as white powder. The crude product was purified via flash chromatography using silica (conditioned with 5 vol\% $\mathrm{NEt}_{3}$ ) and PE/EE (5:1) as eluent. The pure product was obtained as a white powder in $80 \%$ yield. ${ }^{1} \mathrm{H}$ NMR $\left(\mathrm{CDCl}_{3}, 20{ }^{\circ} \mathrm{C}\right): \delta=7.66(\mathrm{br}, 4 \mathrm{H}, \mathrm{Ph}), 7.58$ (br, 4H, Ph), 7.38-7.05 (m, 33H, Ph, py $\left.{ }^{4}\right), 6.06$ (d, $\left.{ }^{3} J_{\mathrm{HH}}=8.0 \mathrm{~Hz}, 2 \mathrm{H}, \mathrm{py}{ }^{3,5}\right), 5.50\left(\mathrm{~d},{ }^{2} J_{\mathrm{PH}}=3.9 \mathrm{~Hz}, 2 \mathrm{H}\right.$, $\mathrm{NH}), 5.26\left(\mathrm{dd},{ }^{3} J_{\mathrm{HH}}=8.4 \mathrm{~Hz},{ }^{4} J_{\mathrm{PH}}=2.8 \mathrm{~Hz}, 2 \mathrm{H}, \mathrm{CH}^{\mathrm{TAD}}\right)$, $4.86\left(\mathrm{~d},{ }^{3} J_{\mathrm{HH}}=8.5 \mathrm{~Hz}, 2 \mathrm{H}, \mathrm{CH}^{\mathrm{TAD}}\right), 1.21\left(\mathrm{~s}, 6 \mathrm{H}, \mathrm{CH}_{3}^{\mathrm{TAD}}\right)$, $0.23\left(\mathrm{~s}, 6 \mathrm{H}, \mathrm{CH}_{3}^{\mathrm{TAD}}\right) \mathrm{ppm} ;{ }^{13} \mathrm{C}\left\{{ }^{1} \mathrm{H}\right\} \mathrm{NMR}\left(\mathrm{CDCl}_{3}, 20{ }^{\circ} \mathrm{C}\right)$ : $\delta=154.36\left(\mathrm{~d},{ }^{2} J_{\mathrm{CP}}=17.3 \mathrm{~Hz}, \mathrm{py}^{2,6}\right), 146.12(\mathrm{Ph}), 145.53$ $\left(\mathrm{d},{ }^{3} J_{\mathrm{CP}}=2.8 \mathrm{~Hz}, \mathrm{Ph}\right), 141.73\left(\mathrm{~d},{ }^{3} J_{\mathrm{CP}}=1.9 \mathrm{~Hz}, \mathrm{Ph}\right)$, $140.95\left(\mathrm{~d},{ }^{3} J_{\mathrm{CP}}=1.8 \mathrm{~Hz}, \mathrm{Ph}\right), 139.22\left(\mathrm{py}^{4}\right), 129.02(\mathrm{Ph})$, $128.59(\mathrm{~d}, J=3.4 \mathrm{~Hz}, \mathrm{Ph}), 128.19(\mathrm{Ph}), 127.80(\mathrm{Ph})$, $127.75(\mathrm{Ph}), 127.53(\mathrm{Ph}), 127.45(\mathrm{Ph}), 127.36(\mathrm{Ph}), 127.26$

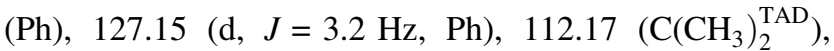
$100.75\left(\mathrm{~d},{ }^{3} J_{\mathrm{CP}}=13.2 \mathrm{~Hz}, \mathrm{py}^{3,5}\right), 82.95\left(\mathrm{C}(\mathrm{Ph})_{2}\right), 82.67(\mathrm{~d}$, $\left.{ }^{3} J_{\mathrm{CP}}=7.7 \mathrm{~Hz}, \mathrm{CH}^{\mathrm{TAD}}\right), 82.48\left(\mathrm{~d},{ }^{3} J_{\mathrm{CP}}=6.0 \mathrm{~Hz}, \mathrm{CH}^{\mathrm{TAD}}\right)$, $82.24\left(\mathrm{C}(\mathrm{Ph})_{2}\right), 27.49\left(\mathrm{CH}_{3}^{\mathrm{TAD}}\right), 25.32\left(\mathrm{CH}_{3}^{\mathrm{TAD}}\right) \mathrm{ppm}$; ${ }^{31} \mathrm{P}\left\{{ }^{1} \mathrm{H}\right\} \mathrm{NMR}\left(\mathrm{CDCl}_{3}, 20{ }^{\circ} \mathrm{C}\right): \delta=134.0 \mathrm{ppm}$.

\section{General synthetic procedure for the synthesis of $\left[\mathrm{M}(\mathrm{PNP}-\mathrm{TADDOL})(\mathrm{CO})_{3}\right]$ complexes}

A suspension of the metal hexacarbonyl $(0.60 \mathrm{mmol})$ and 1 equiv of the PNP ligand $2(0.60 \mathrm{mmol})$ in $4 \mathrm{~cm}^{3} \mathrm{CH}_{3} \mathrm{CN}$ was placed in a $20 \mathrm{~cm}^{3}$ sealed glass tube and stirred for $2 \mathrm{~h}$ at $135{ }^{\circ} \mathrm{C}$, whereupon a clear solution was formed. The reaction mixture was allowed to cool to room temperature without stirring. The products were then obtained as crystalline materials and were decanted and washed with $n$ pentane. The solvent was removed under reduced pressure.

$\left(N^{2}, N^{6}\right.$-bis((3aR,8aR)-2,2-dimethyl-4,4,8,8-tetraphenyltetrahydro[1,3]dioxolo[4,5-e][1,3,2]dioxaphosphepin-6-yl)pyr-

idine-2,6-diamine)(tricarbonyl)chromium(0)

([Cr(PNP-TADDOL)(CO) 3$], 3, \mathrm{C}_{70} \mathrm{H}_{61} \mathrm{CrN}_{3} \mathrm{O}_{11} \mathrm{P}_{2}$ ) The product was obtained as a yellow solid in $91 \%$ yield. ${ }^{1} \mathrm{H}$ NMR
$\left(\mathrm{CD}_{2} \mathrm{Cl}_{2}, 20{ }^{\circ} \mathrm{C}\right): \delta=7.60(\mathrm{~d}, J=4.2 \mathrm{~Hz}, 4 \mathrm{H}, \mathrm{Ph}), 7.46(\mathrm{~d}$, $J=3.3 \mathrm{~Hz}, 4 \mathrm{H}, \mathrm{Ph}), 7.32$ (br, $4 \mathrm{H}, \mathrm{Ph}), 7.25$ (br, 2H, Ph), 7.24-7.15 (m, 26H, Ph), $6.88\left(\mathrm{t}, J=7.4 \mathrm{~Hz}, 1 \mathrm{H}, \mathrm{py}^{4}\right), 5.33$ $\left(\mathrm{dd},{ }^{3} J_{\mathrm{HH}}=27.0 \mathrm{~Hz},{ }^{4} J_{\mathrm{PH}}=7.7 \mathrm{~Hz}, 4 \mathrm{H}, \mathrm{CH}^{\mathrm{TAD}}\right), 5.31(\mathrm{~d}$, $\left.{ }^{3} J_{\mathrm{HH}}=7.7,2 \mathrm{H}, \mathrm{py}^{3,5}\right), 5.11(\mathrm{~s}, 2 \mathrm{H}, \mathrm{NH}), 0.55(\mathrm{~s}, 6 \mathrm{H}$, $\left.\mathrm{CH}_{3}^{\mathrm{TAD}}\right), 0.40$ (s, 6H, $\mathrm{CH}_{3}^{\mathrm{TAD}}$ ) ppm; ${ }^{13} \mathrm{C}\left\{{ }^{1} \mathrm{H}\right\} \mathrm{NMR}\left(\mathrm{CD}_{2-}\right.$ $\left.\mathrm{Cl}_{2}, 20{ }^{\circ} \mathrm{C}\right): \delta=228.0(\mathrm{t}, J=13.4 \mathrm{~Hz}, \mathrm{CO}), 216.9(\mathrm{t}$, $J=20.5 \mathrm{~Hz}, \mathrm{CO}), 156.5\left(\mathrm{t},{ }^{2} J_{\mathrm{CP}}=13.2 \mathrm{~Hz}, \mathrm{py}^{2,6}\right), 144.2$ $(\mathrm{Ph}), 142.1(\mathrm{Ph}), 140.9(\mathrm{Ph}), 140.1(\mathrm{Ph}), 135.9\left(\mathrm{py}^{4}\right), 128.0$ $(\mathrm{Ph}), 127.9(\mathrm{Ph}), 127.8(\mathrm{Ph}), 127.4(\mathrm{~d}, J=5.4 \mathrm{~Hz}, \mathrm{Ph})$, $127.2(\mathrm{Ph}), 126.5(\mathrm{~d}, J=9.6 \mathrm{~Hz}, \mathrm{Ph}), 126.3(\mathrm{Ph}), 126.1$ $(\mathrm{Ph}), 126.0(\mathrm{Ph}), 114.1\left(\mathrm{C}\left(\mathrm{CH}_{3}\right)_{2}^{\mathrm{TAD}}\right), 97.0\left(\mathrm{py}^{3,5}\right), 87.5(\mathrm{t}$, $\left.J=8.5 \mathrm{~Hz}, \mathrm{C}(\mathrm{Ph})_{2}\right), 86.2\left(\mathrm{C}(\mathrm{Ph})_{2}\right), 78.9\left(\mathrm{CH}^{\mathrm{TAD}}\right), 78.4$ $\left(\mathrm{CH}^{\mathrm{TAD}}\right), 25.8\left(\mathrm{CH}_{3}^{\mathrm{TAD}}\right), 25.2\left(\mathrm{CH}_{3}^{\mathrm{TAD}}\right) \mathrm{ppm} ;{ }^{31} \mathrm{P}\left\{{ }^{1} \mathrm{H}\right\}$ NMR $\left(\mathrm{CD}_{2} \mathrm{Cl}_{2}, \quad 20^{\circ} \mathrm{C}\right): \quad \delta=197.8 \mathrm{ppm} ; \quad \mathrm{IR}$ (ATR): $\bar{v}=1974\left(v_{\mathrm{CO}}\right), 1921\left(v_{\mathrm{CO}}\right), 1865\left(v_{\mathrm{CO}}\right) \mathrm{cm}^{-1}$.

$\left(N^{2}, N^{6}\right.$-bis((3aR,8aR)-2,2-dimethyl-4,4,8,8-tetraphenyltetrahydro[1,3]dioxolo[4,5-e][1,3,2]dioxaphosphepin-6-yl)pyridine-2,6-diamine)(tricarbonyl)molybdenum(0) ([Mo(PNP-TADDOL)(CO) 3$], 4, \mathrm{C}_{70} \mathrm{H}_{61} \mathrm{MoN}_{3} \mathrm{O}_{11} \mathrm{P}_{2}$ ) The product was obtained as a yellow solid in $90 \%$ yield. ${ }^{1} \mathrm{H}$ NMR $\left(\mathrm{CD}_{2} \mathrm{Cl}_{2}, 20{ }^{\circ} \mathrm{C}\right): \delta=7.57(\mathrm{~d}, J=7.3 \mathrm{~Hz}, 4 \mathrm{H}, \mathrm{Ph}), 7.44$ 7.39 (m, 4H, Ph), 7.34 (vt, $J=7.4 \mathrm{~Hz}, 4 \mathrm{H}, \mathrm{Ph}), 7.30$ (vt, $J=7.2 \mathrm{~Hz}, 2 \mathrm{H}, \mathrm{Ph}), 7.26-7.11(\mathrm{~m}, 26 \mathrm{H}, \mathrm{Ph}), 6.90(\mathrm{t}$, $\left.J=7.9 \mathrm{~Hz}, 1 \mathrm{H}, \mathrm{py}^{4}\right), 5.34(\mathrm{dd}, J=36.4,7.9 \mathrm{~Hz}, 4 \mathrm{H}$, $\left.\mathrm{CH}^{\mathrm{TAD}}\right), 5.27\left(\mathrm{~d},{ }^{3} J_{\mathrm{HH}}=8.0,2 \mathrm{H}, \mathrm{py}^{3,5}\right), 5.09(\mathrm{~s}, 2 \mathrm{H}, \mathrm{NH})$, $0.50\left(\mathrm{~s}, 6 \mathrm{H}, \mathrm{CH}_{3}^{\mathrm{TAD}}\right), 0.40\left(\mathrm{~s}, 6 \mathrm{H}, \mathrm{CH}_{3}^{\mathrm{TAD}}\right) \mathrm{ppm} ;{ }^{13} \mathrm{C}\left\{{ }^{1} \mathrm{H}\right\}$ NMR $\left(\mathrm{CD}_{2} \mathrm{Cl}_{2}, 20{ }^{\circ} \mathrm{C}\right): \delta=221.6(\mathrm{t}, J=8.1 \mathrm{~Hz}, \mathrm{CO})$, $206.7(\mathrm{t}, J=13.0 \mathrm{~Hz}, \mathrm{CO}), 155.4\left(\mathrm{t}, J=10.3 \mathrm{~Hz}, \mathrm{py}^{2,6}\right)$, $144.2(\mathrm{Ph}), 141.5(\mathrm{Ph}), 140.9(\mathrm{Ph}), 140.0(\mathrm{Ph}), 136.3\left(\mathrm{py}^{4}\right)$, $127.9(\mathrm{Ph}), 127.8(\mathrm{Ph}), 127.6(\mathrm{Ph}), 127.3(\mathrm{~d}, J=12.9 \mathrm{~Hz}$, $\mathrm{Ph}), 127.1(\mathrm{Ph}), 126.4(\mathrm{~d}, J=10.6 \mathrm{~Hz}, \mathrm{Ph}), 126.2(\mathrm{Ph})$, $126.1(\mathrm{Ph}), 126.0(\mathrm{Ph}), 114.2\left(\mathrm{C}\left(\mathrm{CH}_{3}\right)_{2}^{\mathrm{TAD}}\right), 97.6\left(\mathrm{py}^{3,5}\right)$, $87.9\left(\mathrm{t}, \quad J=7.9 \mathrm{~Hz}, \mathrm{C}(\mathrm{Ph})_{2}\right), 85.9\left(\mathrm{C}(\mathrm{Ph})_{2}\right), 78.7 \quad(\mathrm{~d}$, $\left.J=27.6 \mathrm{~Hz}, \mathrm{CH}^{\mathrm{TAD}}\right), 25.7\left(\mathrm{CH}_{3}^{\mathrm{TAD}}\right), 25.3\left(\mathrm{CH}_{3}^{\mathrm{TAD}}\right) \mathrm{ppm}$; ${ }^{31} \mathrm{P}\left\{{ }^{1} \mathrm{H}\right\} \quad \mathrm{NMR} \quad\left(\mathrm{CD}_{2} \mathrm{Cl}_{2}, \quad 20{ }^{\circ} \mathrm{C}\right): \quad \delta=172.7 \mathrm{ppm} ; \quad \mathrm{IR}$ (ATR): $\bar{v}=1980\left(v_{\mathrm{CO}}\right), 1946\left(v_{\mathrm{CO}}\right), 1867\left(v_{\mathrm{CO}}\right) \mathrm{cm}^{-1}$.

$\left(N^{2}, N^{6}\right.$-bis $((3 a R, 8 \mathrm{a} R)-2,2-$ dimethyl-4,4,8,8-tetraphenyltetrahydro[1,3]dioxolo[4,5-e][1,3,2]dioxaphosphepin-6-yl)pyridine-2,6-diamine)(tricarbonyl)tungsten(0)

([W(PNP-TADDOL)(CO) 3$], 5, \mathrm{C}_{70} \mathrm{H}_{61} \mathrm{WN}_{3} \mathrm{O}_{11} \mathrm{P}_{2}$ ) The product was obtained as a yellow solid in $89 \%$ yield. ${ }^{1} \mathrm{H}$ NMR $\left(\mathrm{CD}_{2} \mathrm{Cl}_{2}, 20^{\circ} \mathrm{C}\right): \delta=7.58(\mathrm{~d}, J=7.2 \mathrm{~Hz}, 4 \mathrm{H}, \mathrm{Ph}), 7.42(\mathrm{~d}$, $J=7.5 \mathrm{~Hz}, 4 \mathrm{H}, \mathrm{Ph}), 7.38-7.35(\mathrm{~m}, 4 \mathrm{H}, \mathrm{Ph}), 7.32(\mathrm{~d}$, $J=7.9 \mathrm{~Hz}, 2 \mathrm{H}, \mathrm{Ph}), 7.25-7.16(\mathrm{~m}, 26 \mathrm{H}, \mathrm{Ph}), 6.88(\mathrm{t}$, $\left.J=7.9 \mathrm{~Hz}, 1 \mathrm{H}, \mathrm{py}^{4}\right), 5.35(\mathrm{dd}, J=27.7 \mathrm{~Hz}, 7.9 \mathrm{~Hz}, 4 \mathrm{H}$, $\left.\mathrm{CH}^{\mathrm{TAD}}\right), \quad 5.30\left(\mathrm{~d},{ }^{3} J_{\mathrm{HH}}=8.0, \quad 2 \mathrm{H}, \quad \mathrm{py}^{3,5}\right), \quad 5.09 \quad(\mathrm{~d}$, $\left.{ }^{2} J_{\mathrm{PH}}=8.0 \mathrm{~Hz}, 2 \mathrm{H}, \mathrm{NH}\right), 0.50\left(\mathrm{~s}, 6 \mathrm{H}, \mathrm{CH}_{3}^{\mathrm{TAD}}\right), 0.41(\mathrm{~s}, 6 \mathrm{H}$, $\left.\mathrm{CH}_{3}^{\mathrm{TAD}}\right)$ ppm; ${ }^{13} \mathrm{C}\left\{{ }^{1} \mathrm{H}\right\}$ NMR $\left(\mathrm{CD}_{2} \mathrm{Cl}_{2}, 20{ }^{\circ} \mathrm{C}\right): \delta=214.3$ (br, CO), 201.0 (t, $J=9.3 \mathrm{~Hz}, \mathrm{CO}), 157.4$ (t, $J=10.6 \mathrm{~Hz}$, 
$\left.\mathrm{py}^{2,6}\right), 145.0(\mathrm{Ph}), 142.4(\mathrm{Ph}), 141.9(\mathrm{Ph}), 140.9(\mathrm{Ph}), 137.4$ $\left(\mathrm{py}^{4}\right), 128.8(\mathrm{Ph}), 128.8(\mathrm{Ph}), 128.5(\mathrm{Ph}), 128.4(\mathrm{~d}$, $J=12.2 \mathrm{~Hz}, \mathrm{Ph}), 128.1(\mathrm{Ph}), 127.5(\mathrm{~d}, J=10.3 \mathrm{~Hz}, \mathrm{Ph})$, $127.2(\mathrm{Ph}), 127.1(\mathrm{Ph}), 127.0(\mathrm{Ph}), 115.2\left(\mathrm{C}\left(\mathrm{CH}_{3}\right)_{2}^{\mathrm{TAD}}\right)$, $97.8\left(\mathrm{py}^{3,5}\right), 89.0\left(\mathrm{t}, J=7.9 \mathrm{~Hz}, \mathrm{C}(\mathrm{Ph})_{2}\right), 87.1\left(\mathrm{C}(\mathrm{Ph})_{2}\right)$, $79.7\left(\mathrm{~d}, \quad J=37.2 \mathrm{~Hz}, \quad \mathrm{CH}^{\mathrm{TAD}}\right), \quad 26.7 \quad\left(\mathrm{CH}_{3}^{\mathrm{TAD}}\right), \quad 26.2$ $\left(\mathrm{CH}_{3}^{\mathrm{TAD}}\right) \mathrm{ppm} ;{ }^{31} \mathrm{P}\left\{{ }^{1} \mathrm{H}\right\} \mathrm{NMR}\left(\mathrm{CD}_{2} \mathrm{Cl}_{2}, 20{ }^{\circ} \mathrm{C}\right): \delta=153.8$ $\left({ }^{1} J_{\mathrm{w}-\mathrm{p}}=498.8 \mathrm{~Hz}\right) \mathrm{ppm}$; IR (ATR): $\bar{v}=1978\left(v_{\mathrm{CO}}\right), 1932$ $\left(v_{\mathrm{CO}}\right), 1865\left(v_{\mathrm{CO}}\right) \mathrm{cm}^{-1}$.

\section{X-ray structure determination}

X-ray diffraction data of $\mathbf{4}$, in the form of the methylene chloride solvate $\mathbf{4} \cdot 3 \mathrm{CH}_{2} \mathrm{Cl}_{2}$ (CCDC 1845599), were collected at $T=100 \mathrm{~K}$ in a dry stream of nitrogen on a Bruker Kappa APEX II diffractometer system using graphitemonochromatized Mo $K \alpha$ radiation $(\lambda=0.71073 \AA)$ and fine sliced $\varphi$ - and $\omega$-scans. Data were reduced to intensity values with SAINT and an absorption correction was applied with the multi-scan approach implemented in SADABS [37]. The structure was solved by the dual-space approach implemented in SHELXT [38] and refined against $F^{2}$ with SHELXL [39]. Non-hydrogen atoms were refined anisotropically. The $\mathrm{H}$ atoms connected to $\mathrm{C}$ atoms were placed in calculated positions and thereafter refined as riding on the parent atoms. The amine-hydrogen atoms were located from difference Fourier maps and refined freely. The absolute structure was confirmed by resonant scattering [Flack parameter 0.025(14)]. Important crystallographic data are: $\mathrm{C}_{70} \mathrm{H}_{61} \mathrm{MoN}_{3} \mathrm{O}_{11} \mathrm{P}_{2} 3\left(\mathrm{CH}_{2} \mathrm{Cl}_{2}\right), M_{\mathrm{r}-}$ $=1532.87$, yellow plates, $0.50 \times 0.25 \times 0.04 \mathrm{~mm}$, monoclinic, space group $P 2_{1}$ (no. 4), $a=10.0035(8) \AA$, $b=20.2073(18) \AA, c=18.1231(16) \AA, \beta=103.943(4)^{\circ}$, $V=3555.5(5) \AA^{3}, \quad Z=2, \quad \mu=0.517 \mathrm{~mm}^{-1}, \quad d_{\mathrm{x}}=$ $1.432 \mathrm{~g} \mathrm{~cm}^{-3}$. 20,807 reflections were collected up to $\theta_{\text {max }}=30.0^{\circ} ; \quad R_{1}=0.0548 \quad(14,397$ reflections with $I>2 \sigma(I)), w R_{2}=0.1072$ (all data), 877 parameters.

Acknowledgements Open access funding provided by Austrian Science Fund (FWF). Financial support by the Austrian Science Fund (FWF) is gratefully acknowledged (Project No. P28866-N34). The $\mathrm{X}$-ray Center of the Vienna University of Technology is acknowledged for financial support and for providing access to the singlecrystal diffractometer.

Open Access This article is distributed under the terms of the Creative Commons Attribution 4.0 International License (http://creative commons.org/licenses/by/4.0/), which permits unrestricted use, distribution, and reproduction in any medium, provided you give appropriate credit to the original author(s) and the source, provide a link to the Creative Commons license, and indicate if changes were made.

\section{References}

1. Gossage RA, van de Kuil LA, van Koten G (1998) Acc Chem Res $31: 423$

2. Albrecht M, van Koten G (2001) Angew Chem Int Ed 40:3750

3. van der Boom ME, Milstein D (2003) Chem Rev 103:1759

4. Liang LC (2006) Coord Chem Rev 250:1152

5. Morales-Morales D (2018) Pincer compounds-chemistry and applications. Elsevier, Amsterdam

6. Nishiyama H (2007) Chem Soc Rev $36: 1133$

7. Benito-Garagorri D, Kirchner K (2008) Acc Chem Res 41:201

8. Selander N, Szabo KJ (2011) Chem Rev 111:2048

9. van Koten G, Milstein D (2013) Organometallic pincer chemistry. Topics in organometallic chemistry, vol 40. Springer, Berlin

10. Szabo KJ, Wendt OF (2014) Pincer and pincer-type complexes: applications in organic synthesis and catalysis. Wiley-VCH, Weinheim

11. Younus HA, Ahmad N, Su W, Verpoort F (2014) Coord Chem Rev 276:112

12. Murugesan S, Kirchner K (2016) Dalton Trans 45:416

13. Schirmer W, Flörke U, Haupt HJ (1987) Z Anorg Allg Chem 545:83

14. Lang HF, Fanwick PE, Walton RA (2002) Inorg Chim Acta 329:1

15. Benito-Garagorri D, Becker E, Wiedermann J, Lackner W, Pollak M, Mereiter K, Kisala J, Kirchner K (2006) Organometallics 25:1900

16. Öztopcu Ö, Holzhacker C, Puchberger M, Weil M, Mereiter K, Veiros LF, Kirchner K (2013) Organometallics 32:3042

17. Castro-Rodrigo R, Chakraborty S, Munjanja L, Brennessel WW, Jones WD (2016) Organometallics 35:3124

18. Wingard LA, White PS, Templeton JL (2012) Dalton Trans 41:11438

19. Alzamly A, Gambarotta S, Korobkov I (2013) Organometallics 32:7204

20. Alzamly A, Gambarotta S, Korobkov I (2014) Organometallics 33:1602

21. Hebden TJ, Schrock RR, Takase MK, Mueller P (2012) Chem Commun 48:1851

22. Arashiba K, Kuriyama S, Nakajima K, Nishibayashi Y (2013) Chem Commun 49:11215

23. Kinoshita E, Arashiba K, Kuriyama S, Miyake Y, Shimazaki R, Nakanishi H, Nishibayashi Y (2012) Organometallics 31:8437

24. Arashiba A, Sasaki K, Kuriyama S, Miyake Y, Nakanishi H, Nishibayashi Y (2012) Organometallics 31:2035

25. Arashiba K, Miyake Y, Nishibayashi Y (2011) Nat Chem 3:120

26. Tanaka H, Arashiba K, Kuriyama S, Sasada A, Nakajima K, Yoshizawa K, Nishibayashi Y (2014) Nat Commun 5:1

27. de Aguiar SRMM, Stöger B, Pittenauer E, Allmaier G, Puchberger M, Veiros LF, Kirchner KJ (2014) J Organomet Chem 760:74

28. de Aguiar SRMM, Öztopcu Ö, Stöger B, Mereiter K, Veiros LF, Pittenauer E, Allmaier G, Kirchner K (2014) Dalton Trans 43:14669

29. de Aguiar SRMM, Stöger B, Pittenauer E, Allmaier G, Veiros LF, Kirchner K (2016) Dalton Trans 45:13834

30. de Aguiar SRMM, Öztopcu Ö, Troiani A, de Petris G, Weil M, Pittenauer E, Allmaier G, Veiros LF, Kirchner K (2018) Eur J Inorg Chem 7:876

31. Mastalir M, de Aguiar SRMM, Glatz M, Stöger B, Kirchner K (2016) Organometallics 35:229

32. Gorla F, Venanzi LM, Albinati A (1994) Organometallics 13:43

33. Williams BS, Dani P, Lutz M, Spek AL, van Koten G (2001) Helv Chim Acta 84:3519

34. Morales-Morales D, Cramer RE, Jensen CM (2002) J Organomet Chem 54:44 
35. Perrin DD, Armarego WLF (1988) Purification of laboratory chemicals, 3rd edn. Pergamon, New York

36. Kranich R, Eis K, Geis O, Mühle S, Bats JW, Schmalz HG (2000) Chem Eur J 6:2874
37. Bruker AXS, Inc. (2012) Bruker computer programs: APEX2, SAINT, and SADABS. Bruker AXS, Inc., Madison

38. Sheldrick GM (2015) Acta Crystallogr A 71:3

39. Sheldrick GM (2015) Acta Crystallogr C 71:3 